\title{
Oscilações de 6-7 dias na mesosfera e ionosfera equatorial
}

Romeu Ferreira Castro, DF/UEPB, Brazil

Lourivaldo Mota Lima, DF/UEPB, Brazil

Edvaldo de Oliveira Alves, DF/UEPB, Brazil

Paulo Prado Batista INPE, Brazil

Barclay Robert Clemesha, INPE, Brazil

H. Takahashi, INPE, Brazil

Inez S. Batista, , INPE, Brazil

M. A. Abdu, INPE, Brazil

Copyright 2009, SBGf - Sociedade Brasileira de Geofísica

This paper was prepared for presentation during the $11^{\text {th }}$ International Congress of the Brazilian Geophysical Society held in Salvador, Brazil, August 24-28, 2009.

Contents of this paper were reviewed by the Technical Committee of the $11^{\text {th }}$ International Congress of the Brazilian Geophysical Society and do not necessarily represent any position of the SBGf, its officers or members. Electronic reproduction or storage of any part of this paper for commercial purposes without the written consent of the Brazilian Geophysical Society is prohibited.

\section{Abstract}

Simultaneous 6-7 days oscillations have been observed in mesospheric wind measurements, obtained by meteor radar at São João do Cariri, and in the evening $F$ region vertical plasma drift measured by digisonde at Fortaleza, Brazil. The result suggests that the 6-7 day planetary oscillations presents in the equatorial mesosphere can modulate the ionosphere.

\section{Introdução}

A base da altura virtual da camada $F$ da ionosfera, $h$ ' $F$, é sensível ao campo elétrico local. Na região do equador magnético, onde o campo magnético é horizontal, $O$ efeito de $E^{\prime} ¥ B^{\prime}$ é $O$ principal responsável pelo movimento vertical de $h^{\prime} F$ (Takahashi, et al. 2006). Vários são os fatores que devem ser considerados para estudar a variabilidade do parâmetro ionosférico $h$ ' $F$ durante o anoitecer na região equatorial, dentre os quais temos o caso do transporte do campo elétrico a partir das regiões polares durante condições magneticamente perturbadas, ou ainda a modulação pelo sistema de ventos termosféricos em escala global devido ao aquecimento Joule nas regiões polares. Também existem fenômenos esporádicos relacionados com perturbações magnéticas. Por outro lado, o sistema de ventos termosféricos está sujeito a uma forte oscilação diurna que acompanha o movimento do sol. Adicionalmente, a presença de ondas atmosféricas de escala planetária pode modular os ventos de marés, resultando em variações na altura da base da camada $F$ da ionosfera (Lastovicka, 2006).

O objetivo deste trabalho é estudar as variações na base da altura virtual da camada $F$ da ionosfera, $h$ 'F, na região e nos ventos meteóricos da região equatorial.

\section{Metodologia}

A base da altura virtual da camada $F$ da ionosfera, $h ' F$, é sensível ao campo elétrico local. Na região do equador magnético, onde o campo magnético é horizontal, 0 efeito de $E^{\prime} ¥ B^{\prime}$ é 0 principal responsável pelo movimento vertical de $h$ ' $F$ (Takahashi, et al. 2006). Vários são os fatores que devem ser considerados para estudar a variabilidade do parâmetro ionosférico $h$ ' $F$ durante o anoitecer na região equatorial, dentre os quais temos o caso do transporte do campo elétrico a partir das regiões polares durante condições magneticamente perturbadas, ou ainda a modulação pelo sistema de ventos termosféricos em escala global devido ao aquecimento Joule nas regiões polares. Também existem fenômenos esporádicos relacionados com perturbações magnéticas. Por outro lado, o sistema de ventos termosféricos está sujeito a uma forte oscilação diurna que acompanha o movimento do sol. Adicionalmente, a presença de ondas atmosféricas de escala planetária pode modular os ventos de marés, resultando em variações na altura da base da camada $F$ da ionosfera (Lastovicka, 2006)

Neste estudo foram utilizadas medidas da altura virtual da base da camada $F$ da ionosfera ( $\left.h^{\prime} F\right)$, as quais foram obtidas com uma ionossonda digital instalada em Fortaleza-CE $\left(3,9^{\circ} \mathrm{S}, 38,4^{\circ} \mathrm{O}\right.$, Geomag. $\left.2,1^{\circ} \mathrm{S}\right)$. A ionossonda digital, Digisonde Portable Sounder (DPS-4), é composta por uma antena transmissora, um sistema de antenas receptoras e um transceptor integrado.

Os valores de deriva vertical foram calculados a partir dos dados da Digissonda pela seguinte relação (Abdu et al., 1981):

$$
\mathrm{V}_{\mathrm{z}}=\Delta \mathrm{h}^{\prime} \mathrm{F} / \Delta \mathrm{t}
$$

em que $\Delta \mathrm{e} h^{\prime} F$ é a variação da altura virtual da camada $\mathrm{F}$ e $\Delta t$ é o intervalo de tempo entre duas sondagens sucessivas.

Também foram utilizadas as medidas de vento obtidas através do radar meteórico VHF instalado 
em São João do Cariri-PB $\left(7.4^{\circ} \mathrm{S}, \quad 36.5^{\circ} \mathrm{W}\right)$, denominado de SKiYMET, o qual é um sistema que possui visada de todo o céu, o qual foi projetado para operar em alta repetição de pulsos de freqüências. $O$ sistema usa uma antena transmissora Yagi de três elementos, com 5 antenas receptoras dispostas no solo formando uma cruz assimétrica. Esta configuração interferométrica permite a determinação da trilha meteórica no céu.

As informações fornecidas pelo radar foram utilizadas na estimativa das componentes dos ventos na direção leste-oeste (zonal) e norte-sul (meridional), para intervalos horários sucessivos para 7 camadas atmosféricas com $4 \mathrm{~km}$ de espessura, com uma sobreposição de $0,5 \mathrm{~km}$ na base e na parte superior, centradas em $81,84,87$, $90,93,96$ e $99 \mathrm{~km}$ de altura, tendo sido assumido um vento uniforme e constante em cada altura e intervalo de tempo.

No presente trabalho, os resultados apresentados referem-se a observações obtidas durante o período de setembro a novembro de 2004.

\section{Resultados}

Os dados da altura virtual da base da camada $F$, $h$ ' $F$, obtidos entre $16 \mathrm{~h}$ até as $8 \mathrm{~h}$ do dia seguinte foram usados nas análises. Na Figura 1 estão representados os valores médios da altura virtual da camada $F(h ' F)$ juntamente com os desvios padrão para cada dia do mês de setembro de 2004.

Os resultados mostram que a altura virtual da base da camada $F\left(h^{\prime} F\right)$, em geral, experimenta uma subida após as 16 hs, chamada de pico de préreversão da deriva vertical, que é devida ao campo elétrico na direção leste-oeste gerada pelo dínamo da região $F$.

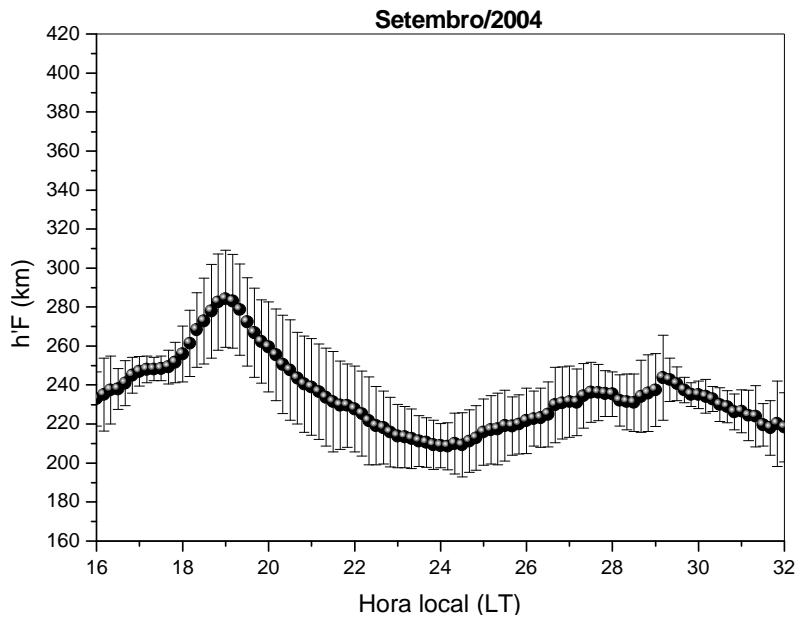

Figura 1 - Valores médios e desvios padrão de h'F observada através da digisonda na camada F sobre Fortaleza, durante o mês de setembro de 2004.
$\mathrm{Na}$ Figura 2 estão representas as velocidades de deriva de h'F, obtidas a partir da variação de $h$ ' $F$ entre 18 e 20 hs (LT), enquanto que na Figura 3, tem-se o espectro de ondaletas do vento zonal sobre S. J. do Cariri, na camada de $93 \mathrm{~km}$ de altitude, referente ao período entre final de agosto e início de dezembro de 2004.

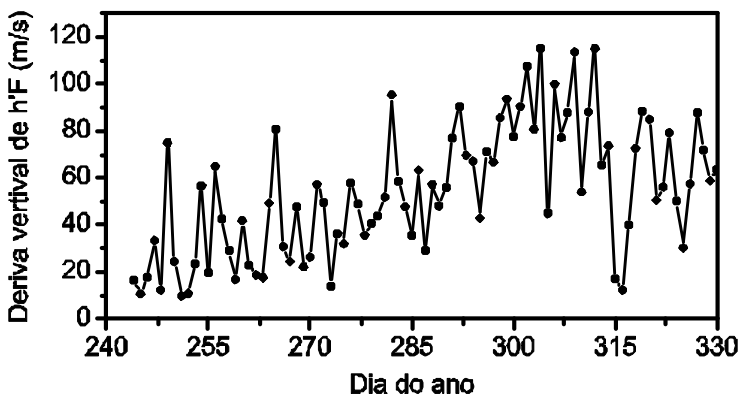

Figura 2 - Velocidade de deriva vertical de h'F (entre 18 e 19 hs) para cada dia durante setembro a novembro de 2004.

Conforme pode ser observado na Figura 2, a velocidade com que a h'F sobe mostra variações dia a dia, conforme mostra o gráfico inferior.

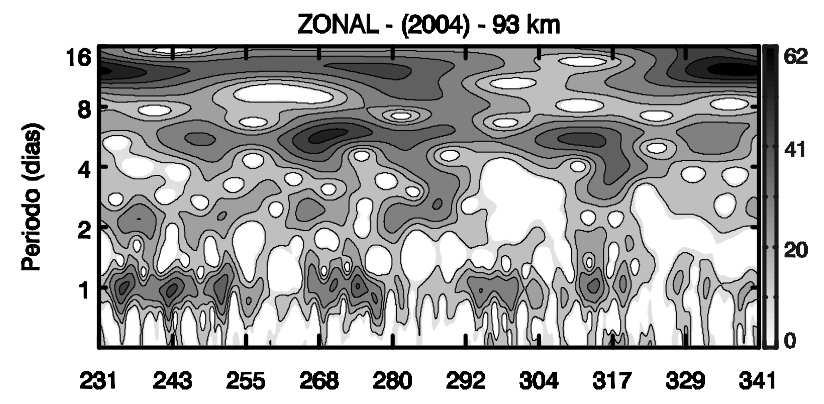

Figura 3 - Espectro de ondaletas do vento zonal sobre S. J. do Cariri, na altitude centrada em $93 \mathrm{~km}$, para o período entre final de agosto e início de dezembro de 2004.

O espectro de ondaletas do vento zonal da região equatorial, apresenta energia espectral que podem ser associadas a várias oscilações, como é o caso da maré diurna, da onda de 2-dias, bem como para ondas de 6-7 dias e com períodos entre 8 e 16 dias

A onda de 6-7 dias é uma importante oscilação, presente na mesosfera durante os meses de outono e primavera (Lima et al., 2005). Para verificar a presença também na ionosfera, os dados de velocidade da velocidade de deriva vertical e do vento zonal foram submetidos a um filtro passa 
banda, na faixa de 5 a 7 dias. Os resultados são apresentados na Figura 4.

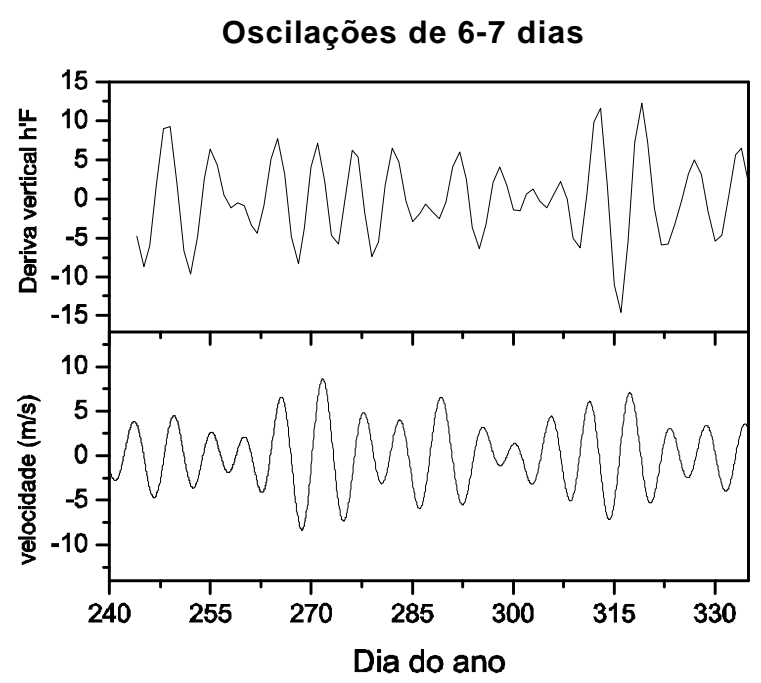

Figura 4 - Comportamento temporal da velocidade de deriva vertical de h'F (Fortaleza) e do vento zonal em $93 \mathrm{~km}$ de altitude (São João do Cariri). Os dados foram filtrados por um filtro passa banda com perídos de corte de 5 e 7 dias.

Da Figura 4 é possível observar que a oscilação de 6-7 dias está presente em ambos os parâmetros, vento mesosférico e altura virtual da base da camada F (h'F), para o período considerado.

Análises da série de valores do índice geomagnético Ap, para o mesmo período, indicam apenas uma forte tempestade magnética entre os dias 08 e 10 de novembro (dias 313-315). Portanto, as oscilações verificadas neste intervalo de dias devem ser vistas com cautela.

Estudo de oscilações associadas com ondas planetárias nos ventos mesosféricos de baixas latitudes e na camada F da ionosfera equatorial, foram conduzidos por Abdu et al. (2006). Os autores sugerem que oscilações de escala planetária observadas no campo de pré-reversão podem se causadas por oscilações nos ventos de marés da região $E$, as quais podem ser moduladas por ondas planetárias que se propagam a partir da baixa e média atmosfera.

Assim, as oscilações de 6-7 dias, observadas nas medidas de vento mesosférico e na velocidade de deriva vertical da altura virtual da base da camada $F$, sugerem que as oscilações de escala planetária de 6-7 dias presentes na mesosfera produzem efeitos de modulação em h'F.

\section{Conclusões}

Observações de oscilações de 6-7 dias presentes quase simultaneamente nas medidas do vento zonal obtidos na região mesosférica equatorial, através de radar meteórico, e na velocidade de deriva da altura virtual da base da camada F (h'F), obtidas de medidas de digisonda em Fortaleza, durante a primavera austral de 2004, sugerem que estas oscilações se propagam na mesosfera e produzem efeitos na ionosfera, em acordo com os estudos de Abdu et. al. (2006) e de Takahashi et al. (2006).

\section{Agradecimentos}

Os autores agradecem ao Instituto Nacional de Pesquisas Espaciais - INPE, ao Observatório de Luminescência Atmosférica da Paraíba - OLAP. Este trabalho foi parcialmente financiado pelo Conselho Nacional de Desenvolvimento Científico e Tecnológico - CNPq, através de bolsa de iniciação científica, e pela Universidade Estadual da Paraíba - UEPB.

\section{Referências}

ABDU, M. A.; BATISTA, I. S.; BITTENCOURT, J. A. Some characteristics of spread $F$ at magnetic equatorial station Fortaleza. Journal of Geophysical Research, V. 86, n.A8, p. 6838-6842, 1981.

ABDU, M. A.; BATISTA, P. P. ; BATISTA, I. S .; BRUM, C. G. M. CARRASCO, A. J E B. REINISCH, W. Planetary wave oscillations in mesospheric winds, equatorial evening prereversal electric field and spread F. Geophys. Research Letters, Vol. 33, L07107, doi:10.1029/2005GL024837, 2006.

LASTOVICKA, J. Forcing of the ionosphere by waves from below, J. Atmos. Solar-Terr. Phys., 68, 479-497, 2006.

LIMA, L. M.; BATISTA, P. P.; CLEMESHA, B. R.; TAKAHASHI, $\mathrm{H}$. The 6.5-day Oscillations Observed in Meteor Winds over Cachoeira Paulista $\left(22.7^{\circ} \mathrm{S}\right)$. Advances in Space Research, 36 (11), p. 2212-2217, 2005.

TAKAHASHI, H.; WRASSE, C. M.; PANCHEVA, D.; ABDU, M. A.; BATISTA, I. S.; LIMA, L. M.; BATISTA, P. P.; CLEMESHA, B. R.; SHIOKAWA, K. Signatures of 3-6 day planetary waves in the equatorial mesosphere and ionosphere. Ann. Geophys., 24, 3343-3350, 2006. 